\title{
Editorial
}

\section{Imaging the heart in adult congenital heart disease}

Over the past two decades most congenital cardiac lesions have become amenable to surgical repair. The trend is to perform more anatomical types of repair at an earlier age. In addition, older patients, who were previously only palliated, present for corrective surgical interventions. Surgical repair is seldom curative and patients will generally require close follow up throughout life. ${ }^{12}$ The total number of adolescent and adult patients with congenital heart disease is steadily increasing and will peak over the next decade. This poses great demands for the provision of dedicated clinical services, which depend on sophisticated imaging techniques.

\section{Common pitfalls}

The use of any imaging method in the assessment of congenital heart disease has to be carefully planned. Investigations should be carried out along the standardised segmental approach used to describe congenital cardiac lesions. ${ }^{3}$ The results obtained by the various techniques are operator dependent. Frequently, incomplete or unsatisfactory information is acquired. This is commonly a result of poor understanding of the underlying anatomy (congenital or postsurgical), misinterpretation of the findings, or poor image information, either because of the wrong choice of technique used or because of technical problems.

Ideally studies should be undertaken only with detailed knowledge of the underlying anatomy; the most likely residual or acquired lesions should be excluded or identified; the information required for any potential surgical intervention obtained; and the strengths and limitations of the imaging technique used understood. Thus, detailed imaging studies in adults with congenital heart disease often benefit from multidisciplinary cooperation.

\section{Techniques \\ RADIOGRAPHY}

Although the information to be obtained from a plain chest $x$ ray is limited it remains one of the most useful imaging techniques in the serial evaluation of heart size and pulmonary vascularity. The cardiothoracic ratio is still the most accurate predictor of exercise capacity after repair of tetralogy of Fallot.

\section{ULTRASOUND}

Transthoracic ultrasound combines many of the advantages of an ideal imaging technique for congenital heart disease. Not only is it non-invasive and universally available, but it can provide detailed and quantifiable information on intracardiac morphology and function. However, in adult patients, in particular following median sternotomy or in patients with severe chest deformities or lung disease, cross sectional image quality often is limited. Despite these limitations, pulsed and continuous wave Doppler interrogation is a very sensitive tool in the serial assessment of haemodynamic lesions such as outflow tract obstruction.

Transoesophageal ultrasound has proved to be of great additional value in patients with poor precordial windows, particularly since high resolution multiplane transducers have become available. The maximum value from transoesophageal ultrasound studies can be obtained in the assessment of lesions and surgical repairs involving the venous return, the atrial chambers, the atrioventricular valves, and the left ventricular outflow tract. ${ }^{45}$ In contrast, information on the ventricular chambers, the right ventricular outflow tract, and the pulmonary arteries is strictly limited. Transoesophageal ultrasound has become an integral part of the monitoring of selected interventional procedures, namely device closure of both atrial and ventricular septal defects. ${ }^{6} 7$

The recent advent of three dimensional reconstruction of ultrasound images will further enhance ultrasound assessment of congenital heart disease. However, in the adult patient with poor transthoracic ultrasound windows, this technique will almost certainly rely entirely on the transoesophageal approach. Currently it appears that there are insurmountable problems in respect of the ultrasonic measurement of right ventricular size and function. Intravascular ultrasound imaging appears to be of value in the assessment of complex right ventricular outflow obstruction, conduits, the pulmonary arteries, and the aortic arch. In these areas it may become a valuable adjunct to angiography and in the monitoring of interventional cardiac catheterisation. Finally, tissue velocity imaging of the myocardium may yield new insights into myocardial structure and function in patients with chronic cyanosis, cardiomyopathies, following cardiac transplantation, and after surgical repair involving the coronary arteries (the arterial switch procedure).

MAGNETIC RESONANCE IMAGING

Despite its persistently limited availability, magnetic resonance imaging has gained an established role in the morphological and functional assessment of adult congenital heart disease. Numerous comparative studies have clearly defined its weaknesses and strengths compared with, in particular, transoesophageal ultrasound, and have documented its superiority in the assessment of ventricular anatomy, size, and function. ${ }^{8} 9$ In addition, its meticulous use in the assessment of the anatomy of pulmonary blood supply and aortic arch anatomy has been shown to be of great additional value to angiography. ${ }^{10} \mathrm{~A}$ closer cooperation between radiologists and cardiologists is likely to further strengthen its clinical role as a primary imaging modality in adult congenital heart disease.

ANGIOGRAPHY

In the practice of paediatric cardiology the role for diagnostic cardiac catheterisation has declined steadily with the rise of cardiac ultrasound. Currently, about $50 \%$ of all cardiac catheterisation procedures in children with congenital heart disease are interventional procedures. The primary aim for diagnostic cardiac catheterisation is shifting towards an accurate assessment of haemodynamics or pulmonary blood supply. These trends should be mirrored in the indications for cardiac catheterisation in adolescent and adult patients with congenital heart disease. Diagnostic studies should only be undertaken once all other imaging techniques have been used to their best effect and clearly defined questions have been raised. In view of the problems and limitations of repeat cardiac catheterisation in patients with congenital heart disease this point attains even greater importance. Numerous patients will have had 
bilateral femoral cutdowns during cardiac catheterisation in childhood and thus vascular access is frequently limited.

In all patients with significant lesions requiring either surgical or catheter intervention, angiography should be considered an integral part of the overall assessment. The principal questions to be answered include an accurate assessment of the haemodynamic lesions, the pulmonary artery anatomy, pressures, and blood flow. In patients older than 30 years and in all those in whom future or past surgery has involved the coronary arteries, selective coronary angiography should be a routine part of the investigation. In contrast, greater utilisation of magnetic resonance imaging should limit, if not obviate, the need for standard ventriculography.

Both diagnostic and interventional cardiac catheterisation in adult patients with congenital heart disease pose specific technical challenges that have to be met by: the provision of the complete range of diagnostic and interventional catheters, occlusion, embolisation, and retrieval devices; large experience of the operator for both diagnostic and interventional catheterisation in congenital heart disease; biplane angiographic equipment and unrestricted procedure time. In addition, because of the high incidence of arrhythmias (particularly postoperative) in these patients, there should be experience and facilities to conduct concomitant electrophysiological studies and radiofrequency ablations. These high requirements can only be met in selected centres.

OTHER IMAGING TECHNIQUES

Other imaging modalities that play an important role in the assessment of adult congenital heart disease, but are currently underused, include radionuclide studies (for the assessment of ventricular size, function, and myocardial perfusion), lung perfusion scintigraphy (in the assessment of pulmonary blood supply) and computed tomography (CT). Spiral CT is likely to enhance the assessment of the aortic arch and the pulmonary vascular supply. It may become the imaging technique of first choice in the assess- ment of patients with complex forms of pulmonary atresia, ventricular septal defect, and major aortopulmonary collateral arteries, despite its high radiation exposure.

\section{Conclusions}

Cardiac imaging in the adult patient with congenital heart disease poses very specific challenges, which can only be met by close multidisciplinary cooperation and the establishment of dedicated centres that have access to and experience with the wide range of imaging modalities. Future challenges include a better understanding of right ventricular morphology and function, and a more functional assessment of congenital or postsurgical cardiac lesions.

OLIVER STUMPER

Consultant Paediatric Cardiologist,

Birmingham Children's Hospital NHS Trust,

Steelhouse Lane,

Birmingham B4 6NH, UK

1 Stark J. Do we really correct congenital heart disease? f Thorac Cardiovasc Surg 1989;97:1-9.

2 Kaplan S. Congenital heart disease in adolescents and adults-natural and postoperative history across age groups. Cardiology Clinics 1993;11:543-56.

Tynan MJ, Becker AE, Macartney FJ, et al. Nomenclature and classification Tynan MJ, Becker AE, Macartney FJ, et al. Nomenclature

4 Sreeram N, Sutherland GR, Geuskens R, et al. The role of transoesophageal Sreeram N, Sutherland GR, Geuskens R, et al. The role of transoesophageal echocardiography in adolescent

5 Marelli AJ, Child JS, Perloff JK. Transesophageal echocardiography in congenital heart disease in the adult. Cardiology Clinics 1993;11:505-20.

6 Hellenbrand WE, Fahey JT, McGowan FX, et al. Transesophageal echocardiographic guidance of transcatheter closure of atrial septal defect. Am $\mathcal{F}$ Cardiol 1990;66:207-13.

7 Stümper O, Sutherland GR, eds. Transesophageal echocardiography in congenital heart disease. London: Edward Arnold, 1994

8 Hirsch R, Kilner PJ, Connelly MS, et al. Diagnosis in adolescents and adults with congenital heart disease. Prospective assessment of individual and combined roles of magnetic resonance imaging and transesophageal echocardiography. Circulation 1994;90:2937-51.

9 Hoppe UC, Dederichs B, Deutsch H, et al. Congenital heart disease in adults and adolescents: comparative value of transthoracic and transesophageal echocardiography and MR imaging. Radiology 1996;199:66977.

10 Kersting-Sommerhoff B, Sechtem U, Higgins CB. Evaluation of pulmonary blood supply by nuclear magnetic resonance imaging in patients with pulmonary atresia. $\mathcal{F}$ Am Coll Cardiol 1988;11:166-71. 